OPEN ACCESS

Edited by:

Michel Denis,

UPR 3251 Laboratoire d'Informatique

pour la Mécanique et les Sciences de l'Ingénieur (LIMSI), France

Reviewed by:

Roland Maurer,

Université de Genève, Switzerland

Michael Hornberger,

University of East Anglia,

United Kingdom

*Correspondence:

Maddalena Boccia

m.boccia@hsantalucia.it;

maddalena.boccia@uniroma1.it

Specialty section:

This article was submitted to

Cognitive Neuroscience,

a section of the journal

Frontiers in Human Neuroscience

Received: 27 May 2019 Accepted: 02 September 2019 Published: 20 September 2019

Citation:

Boccia M, Bonavita A, Diana S, Di Vita A, Ciurli MP and Guariglia C (2019) Topographical Disorientation: Clinical and Theoretical Significance of Long-Lasting Improvements

Following Imagery-Based Training.

Front. Hum. Neurosci. 13:322.

doi: 10.3389/fnhum.2019.00322

\section{Topographical Disorientation: Clinical and Theoretical Significance of Long-Lasting Improvements Following Imagery-Based Training}

\author{
Maddalena Boccia ${ }^{1 *}$, Alessia Bonavita ${ }^{2}$, Sofia Diana ${ }^{1,2,3}$, Antonella Di Vita ${ }^{1,3}$, \\ Maria Paola Ciurli ${ }^{4}$ and Cecilia Guariglia ${ }^{1,2}$
}

${ }^{1}$ Cognitive and Motor Rehabilitation and Neuroimaging Unit, IRCCS Fondazione Santa Lucia, Rome, Italy, ${ }^{2}$ Department of Psychology, "Sapienza" University of Rome, Rome, Italy, ${ }^{3}$ Department of Human Neuroscience, "Sapienza" University of Rome, Rome, Italy, ${ }^{4}$ Neuropsychology Unit, IRCCS Fondazione Santa Lucia, Rome, Italy

Neuropsychological studies on acquired topographical disorientation have provided useful insights into the contribution of different brain regions to human navigation. However, little is known about the possibility to restore navigational skills after brain damage. Here we describe the case of No Longer Lost (NLL), a 49-year-old man who complained of severe topographical disorientation following traumatic brain injury. Extensive neuropsychological evaluation at baseline revealed selective episodic memory deficits and topographical disorientation. NLL underwent 8-week imagerybased treatment (IBT) inspired by current cognitive models of human spatial navigation. After IBT, NLL improved topographical skills and episodic memory. From a clinical point of view, the present study describes a model-based intervention for topographical disorientation. From a theoretical point of view, it provides new insights into the cognitive models of human spatial navigation and straightforward evidence about common phylogenetic roots of brain mechanisms devoted to spatial navigation and memory.

Keywords: acquired topographical disorientation, brain damage, spatial navigation, topographical memory, episodic memory

\section{BACKGROUND}

To orient themselves within the environmental space - namely, the space beyond the sensory horizon (Wolbers and Wiener, 2014) - individuals have to process "online" information about their own position and facing direction (Sulpizio et al., 2017, 2018), as well as to recall previously acquired "offline" information about the environment (Wolbers and Hegarty, 2010). Physiological evidence suggests that "online" information about position and facing direction is coded by distinct neural populations, namely by hippocampal place cells and head-direction cells, respectively (O'Keefe and Dostrovsky, 1971; Taube, 1998; Ekstrom et al., 2003; Vass and Epstein, 2013; Sulpizio et al., 2014). "Offline" environmental knowledge may be represented as Landmark, Route, and Survey representation (Siegel and White, 1975). Landmark representation roughly corresponds to the figurative memory of environmental objects, through which individuals "beacon" toward salient landmarks. Route representation concerns the memory of paths connecting landmarks and is organized according to an egocentric frame of reference. Survey representation implies the encoding of directions and distances between landmarks regardless of the individual's position, 
resembling a map-like representation of the environment. Recent neuroimaging evidence supports this model (Boccia et al., 2016).

Following the BBB model (Byrne et al., 2007), neural populations within the posterior parietal lobe - namely, "egocentric parietal window" (p. 345) - maintain the headcentered egocentric map of the space and provide exclusive access into long-term navigational memory stored in the medial temporal lobe; in the medial temporal lobe, the parahippocampal gyrus develops allocentric (survey) representations and the hippocampus stores long-term spatial memories; finally, a transformation circuit in the retrosplenial cortex allows to transform the spatial representations stored in an allocentric format into an egocentric (route) format, and vice versa. Evidence in nonhuman primates (Kravitz et al., 2011) and humans (Boccia et al., 2014, 2017a), and neuropsychological studies (Aguirre and D'Esposito, 1999) support this model. Specifically, lesions of the posterior parietal cortex yield to egocentric disorientation, namely a deficit in representing the location of objects with respect to the self (Holmes and Horrax, 1919; Levine et al., 1985; Stark et al., 1996). Instead, lesions of the retrosplenial cortex lead to heading disorientation: patients lose the sense of direction and are unable to direct toward locations beyond the vista space (Takahashi et al., 1997), namely the space that can be explored at a glance (Montello, 1993; Wolbers and Wiener, 2014). Landmark recognition deficits are widely reported following lesions in the lingual gyrus: patients fail in recognizing and representing salient environmental stimuli, even in absence of perceptual deficits (Aguirre and D'Esposito, 1999). These neuropsychological findings provide important information about the unique and causal contribution of each specific node of the parieto-medial temporal lobe network of spatial navigation in humans. However, evidence for a redundant code within this network (Ekstrom et al., 2017; Boccia et al., 2019) suggests the possibility that, in presence of a lesion in a specific node, compensation mechanisms may allow the recovery of the impaired process.

Here we describe the case of NLL (acronym for No Longer Lost), a patient with acquired topographical disorientation, who underwent a novel imagery-based neuropsychological rehabilitation for spatial navigation, tailored on the patient's neuropsychological profile and the theoretical framework described above.

\section{CASE PRESENTATION}

NLL, a 49-year-old man working as a realtor, suffered from an extensive head trauma (including facial trauma) and coma, which lasted about 1 week, following a motor vehicle accident. NLL referred to the IRCCS Santa Lucia in Rome 4 years after his accident due to persistent topographical disorientation, even in highly familiar environments, and memory deficits. The CT performed immediately after the event, and the MRI performed 4 years later, revealed a lesion of the right temporal lobe extending also to subcortical areas (Supplementary Figure 1). No neuropsychological evaluation has been performed immediately after the accident or in the following 4 years.
NLL reported that topographical disorientation greatly impacted over his life and professional duties, and that soon after the event he realized he was unable to recognize landmarks and routes, and adopted several compensatory strategies (e.g., he wrote little numbers on the outside wall of the houses he had to visit with customers, he mentally counted the number of bus stops he had to pass to get to the correct place). Also, he reported to be unable to imagine landmarks and routes connecting them, in both familiar and novel environments. Furthermore, he reported episodic memory deficits: he was impaired in recalling events, including, for example, the customers he met or the houses he visited; he was unable to retrieve memories about previous travels reporting that "it is as if I've never been there ... There is no trace about that in my memory." He also reported to be unable to talk about relevant daily events, for example with his father, when he called NLL in the evening, because he could not recall them.

The study was designed in accordance with the principles of the Declaration of Helsinki and was approved by the ethical committee of the IRCCS Fondazione Santa Lucia. Informed consent was obtained from the patient.

\section{Neuropsychological Assessment at Baseline \\ General Neuropsychological Assessment}

NLL performed well within the normal range in tests assessing attention, intelligence, executive functions, language, and working memory (Table 1). He was impaired in tests evaluating visuo-spatial learning and delayed recall (Corsi Block Tapping Test, CBT; Corsi, 1972; Table 2) and visual, spatial, and verbal memory in ecological contexts (RBMT-3; Wilson et al., 2008; Table 3).

\section{Assessment of Navigational Abilities}

Navigational abilities were tested using the DiViNa Developmental Topographical Disorientation Battery (DDTDB), which is extensively described in previous works (Bianchini et al., 2010, 2014; Palermo et al., 2014) and is based on theoretical models of human navigation (Siegel and White, 1975; Wang and Spelke, 2002). NLL's scores were compared with those of men in our database or previous published studies (Table 2), by using $t$-test modified procedure (Singlims.exe; Crawford and Garthwaite, 2002). Age and education were included as covariates (BTD_Cov.exe; Crawford et al., 2011) when necessary.

Walking Corsi Test (WalCT; Piccardi et al., 2008, 2013) Both short-term and long-term memory in navigational vista space (i.e., learning and delayed recall) were assessed. NLL performed as well as controls in short-term memory and delayed recall. However, he was impaired in learning spatial positions (Table 2).

\section{Cognitive Map Test (CMT; laria et al., 2007)}

We assessed both the formation/learning (CMT-L) and the recall (CMT-R) of a cognitive map, asking NLL to travel in a virtual city, in which there were six landmarks (i.e., a cinema, a restaurant, a bar, a hotel, a pharmacy, and a flower shop) (for full description 
TABLE 1 | General neuropsychological assessment.

\begin{tabular}{|c|c|c|}
\hline Test & $\begin{array}{l}\text { NLL's score } \\
\text { (baseline) }\end{array}$ & $\mathrm{ES}^{\mathrm{a}} / \mathrm{PR}^{\mathrm{b}} / \mathrm{SS}^{\mathrm{c}}$ \\
\hline
\end{tabular}

\section{Attention}

Trail making test

$\begin{array}{lcc}\text { A } & 55 \mathrm{~s} & 2^{\mathrm{a}} \\ \mathrm{B} & 90 \mathrm{~s} & 4^{\mathrm{a}} \\ \mathrm{B}-\mathrm{A} & 35 \mathrm{~s} & 4^{\mathrm{a}} \\ \text { Stroop } & & \\ \text { Errors } & 0 & 4^{\mathrm{a}} \\ \text { Time } & 14 & 4^{\mathrm{a}} \\ \text { TEA } & & \\ \text { Alertness - tonic } & 298 & 10^{\mathrm{b}} \\ \quad \text { Phasic } & 237 & 38^{\mathrm{b}} \\ \quad \text { Median } & 0.239 & 99^{\mathrm{b}} \\ \text { Go-NoGo median } & 543 & 42^{\mathrm{b}} \\ \text { False alarms } & 0 & >46^{\mathrm{b}} \\ \text { Divided attention median } & 740 & 12^{\mathrm{b}} \\ \quad \text { Omissions } & \mathbf{5} & \mathbf{4}^{\mathrm{b}} \\ \text { False alarms } & \mathbf{2} & \\ \text { Working memory median } & 684 & 28^{\mathrm{b}} \\ \quad \text { Omissions } & 3 & <16^{\mathrm{b}} \\ \text { False alarms } & 0 & >76^{\mathrm{b}}\end{array}$

PASAT

Stimulus interval $\mathbf{3 0 0 0}$

\begin{tabular}{|c|c|c|c|}
\hline Correct answers & 46 & & 44.2 \\
\hline Errors & 7 & & \\
\hline Omissions & 7 & & \\
\hline Percentage of errors & 23 & & \\
\hline \multicolumn{4}{|l|}{ Stimulus interval 2600} \\
\hline Correct answers & 47 & & 38.69 \\
\hline Errors & 6 & & \\
\hline Omissions & 7 & & \\
\hline Percentage of errors & 22 & & \\
\hline \multicolumn{4}{|l|}{ Stimulus interval 2200} \\
\hline Correct answers & 47 & & 34.77 \\
\hline Errors & 3 & & \\
\hline Omissions & 10 & & \\
\hline Percentage of errors & 22 & & \\
\hline \multicolumn{4}{|l|}{ Stimulus interval 1800} \\
\hline Correct answers & 37 & & 28.07 \\
\hline Errors & 0 & & \\
\hline Omissions & 23 & & \\
\hline Percentage of errors & 38 & & \\
\hline \multicolumn{4}{|l|}{ Intelligence } \\
\hline Raven progressive matrices & 36 & $4^{\mathrm{a}}$ & \\
\hline \multicolumn{4}{|l|}{ Executive functions } \\
\hline \multicolumn{4}{|l|}{ Wisconsin card sorting test } \\
\hline Errors (\%) & 15 & $61^{\mathrm{b}}$ & \\
\hline Perseverative errors (\%) & 8 & $63^{b}$ & \\
\hline Non-perseverative errors (\%) & 7 & $58^{\mathrm{b}}$ & \\
\hline Categories completed & 6 & $>16^{b}$ & \\
\hline
\end{tabular}

(Continued)
TABLE 1 | Continued

\begin{tabular}{|c|c|c|}
\hline Test & $\begin{array}{l}\text { NLL's score } \\
\text { (baseline) }\end{array}$ & $\mathrm{ES}^{\mathrm{a}} / \mathrm{PR}^{\mathrm{b}} / \mathrm{SS}^{\mathrm{c}}$ \\
\hline
\end{tabular}

$\begin{array}{lcc}\begin{array}{l}\text { Number of attempts on } \\ \text { the first category }\end{array} & 12 & >16^{\mathrm{b}} \\ \text { Inability to maintain the set } & 0 & >16^{\mathrm{b}} \\ \text { Learning to learn } & + & >16^{\mathrm{b}} \\ \text { Tower of London } & & \\ \text { Correct responses (total) } & 7 & 118^{\mathrm{c}} \\ \text { Moves (total) } & 8 & 120^{\mathrm{C}} \\ \text { Planning time (total) } & 88 & 114^{\mathrm{c}} \\ \text { Execution time (total) } & 175 & 106^{\mathrm{c}} \\ \text { Total time } & 263 & 100^{\mathrm{c}} \\ \text { Time-limit breaks } & 0 & 104^{\mathrm{c}} \\ \text { Rule breaks } & 0 & 108^{\mathrm{C}}\end{array}$

Language

Fluency

Phonemic

Semantic

Alternate

Shifting

Memory

Digit span (forward)

Rey auditory verbal learning test Immediate recall

Delayed recall

Recognition

Rey-Osterrieth figure

Copy

Immediate recall

Delayed recall

Oblivion

SMIRNI

Words

Buildings

Faces

Space and object perception

VOSP

$\begin{array}{lll}\text { Screening } & 20 & 15 \\ \text { Incomplete letters } & 20 & 17 \\ \text { Silhouettes } & 26 & 16 \\ \text { Objects decision } & 19 & 15 \\ \text { Progressive silhouettes } & \mathbf{1 3} & \mathbf{1 4} \\ \text { Dot counting } & 10 & 8 \\ \text { Position discrimination } & 20 & 8 \\ \text { Number of location } & 10 & 7 \\ \text { Cube analysis } & 10 & 6\end{array}$

Tests are standardized for use with Italian-speaking individuals. Pathological scores are marked in bold. ${ }^{a}$ Equivalent scores adjusted for sex, age, and years of education. ${ }^{b}$ Percentile rank. Performances below the 5 th percentile and equivalent score equal to zero should be considered pathological. "Standard scores; performances below 84 should be considered pathological. 
of the task and virtual environment, see Iaria et al., 2007). NLL performed worse than controls on CMT-L, but comparably to controls on CMT-R (Table 2).

\section{Route Strategy and Landmark Recognition}

Experimenter shows a path in real environmental space, asking the participant to pay attention to all environmental objects. Immediately after, the participant is blindfolded and brought back to the starting point; then she/he is asked to discriminate among distractors $(N=8)$ the specific landmarks $(N=8)$ encountered along the path (maximum score $=16$ ). Finally, the participant has to reproduce the path shown by the examiner (for similar procedure, see Conson et al., 2018). The score is calculated by summing the segments correctly retraced (maximum score $=7$ ). NLL correctly performed landmark recognition (Table 2) and retraced the route without errors (Table 2), even though he claimed he had no clear navigational goal and was unable to imagine the successive steps of the path.

\section{Throwback to the Starting Point}

Participant is asked to go back to the starting point of a route previously shown by the examiner. The score corresponds to the number of segments correctly performed (maximum score $=6$ ). NLL's performance was errorless.

\section{Map-Following Task}

The participant is provided with a map of a real environmental navigational space on which a starting point and a goal are depicted, and asked to draw the shorter path to reach the goal. Then the participant is brought to the starting point and asked to use the map to reach the goal, following the path he/she drew. The score corresponds to the number of segments correctly performed following the path participant drew (maximum score $=4$ ). NLL hesitated at crossroads, but his performance ( $3 / 4$ correct segments) did not differ from that of controls (Table 2), and he correctly recognized his errors and difficulties.

\section{Mental Imagery Skills}

NLL complained of deficits in imaging landmarks and routes; thus, we tested his mental imagery skills by using the Complete Visual Mental Imagery Battery (CVMIB) (Palermo et al., 2016), which allows to assess systematically the ability to generate, maintain, inspect, and transform visual mental images (for a complete description of tests and normative data, see Palermo et al., 2016). For each subtest (Buildings, Objects, Color 1, Color 2, and Color 3, Inspection of Objects and Letters, Folding, and the Mental Rotation), the sum of the correct answers was computed (Table 4). NLL performed worse than controls on Object and Color 2 subtests (Table 4); he performed within the normal range in the remaining subtests (Table 4). Also, Road Map Test (RMT; Money et al., 1965) and 3D Mental Rotation Task (MRT; Thurstone, 1937) were performed comparably to controls.

\section{Intervention Hypothesis}

The neuropsychological assessment revealed a specific deficit in the formation of the cognitive map. Cognitive maps are pivotal for spatial orientation, since they allow individuals to reach any destination within the environmental space. Individuals unable to form a cognitive map get lost more frequently than individuals who are able to form and use cognitive maps of the environment (Iaria and Barton, 2010). Based on NLL's complaints, as well as on evidence on the pivotal role of Mental Imagery in environmental navigation, we submitted him to an imagery-based treatment (IBT), tailored on NLL's difficulties and aimed at improving and restoring navigational skills, with possible generalization to the episodic memory domain. The IBT was inspired by the imagery-based intervention proposed by Kaschel et al. (2002) for memory rehabilitation. Evidence from current cognitive models of spatial navigation and psychophysiological findings were integrated in the IBT (Siegel and White, 1975; Wang and Spelke, 2002; Wolbers and Wiener, 2014). Specifically, IBT followed the developmental hierarchical model proposed by Siegel and White (1975), neurophysiological evidence about head-direction and place coding (O'Keefe and Dostrovsky, 1971; Taube, 1998) and evidence about the impact of the spatial scale (Wolbers and Wiener, 2014). Thus, we developed training activities allowing to progressively move from the figurative memory of landmark representation in the vista space, to the egocentric routebased and allocentric survey-based representation of the environmental space, retracing ontogenetic acquisition stages proposed by Siegel and White (1975). IBT is extensively described in the Supplementary Material. In brief, in a first phase NLL was motivated to the imagery training and acquired the ability to rapidly generate mental images. Then, he underwent a second phase during which he was asked to generate and retrieve navigational mental images of landmarks, routes, and environmental map-like representations (i.e., survey representations).

\section{Post-treatment Neuropsychological Assessment}

After 8 weeks of treatment, the tests in which NLL performed worse than controls were repeated. For all navigational, spatial, and memory tests, alternate versions were used. The only test for which alternate versions were not available is the CVMIB. However, no feedback is provided to participants during the execution of the test and possible test/re-test effects are unlikely. Effectiveness, namely the measure reflecting the potential improvement achievable during rehabilitation, was calculated for each test, as it follows:

$$
\text { Effectiveness }=\frac{\text { Post treament score }- \text { Baseline score }}{\text { Maximum score }- \text { Baseline score }} * 100
$$

Learning of spatial positions within reaching and navigational vista spaces significantly improved, with an effectiveness of 89.79 and $82.97 \%$ on the CBT and the WalCT, respectively; after IBT performances on both tests were comparable with those of the control group (Table 2). Also, after IBT NLL did not differ from the control group on an alternate version of the CMT (Table 2). His performance on an 
TABLE 2 | Performances on DDTDB.

\begin{tabular}{|c|c|c|c|c|c|c|}
\hline & \multicolumn{2}{|c|}{ Baseline } & \multicolumn{2}{|c|}{ Post-treatment } & \multicolumn{2}{|c|}{ Follow-up } \\
\hline & NLL's score & Crawford analysis & NLL's score & Crawford analysis & NLL's score & Crawford analysis \\
\hline Span & $5^{\mathrm{a}}$ & $t=0.047, p=0.481^{*}$ & & & & \\
\hline Learning & $46^{a}$ & $t=-3.187, p=0.001^{*}$ & $134^{a}$ & $t=1.065, p=0.146^{*}$ & $127^{\mathrm{a}}$ & $t=0.727, p=0.235^{*}$ \\
\hline Delayed recall & $3^{a}$ & $t=-1.681, p=0.050^{*}$ & $6^{a}$ & $t=-0.280, p=0.390^{*}$ & $8^{a}$ & $t=0.654, p=0.258^{*}$ \\
\hline Learning & $50^{a}$ & $t=-3.995, p<0.001^{*}$ & $128^{\mathrm{a}}$ & $t=0.582, p=0.282^{*}$ & $101^{a}$ & $t=-1.002, p=0.161^{*}$ \\
\hline Delayed recall & $8^{a}$ & $t=0.412, p=0.341^{*}$ & $6^{a}$ & $t=-1.650, p=0.053^{*}$ & $8^{a}$ & $t=0.412, p=0.341^{*}$ \\
\hline \multicolumn{7}{|c|}{ Cognitive map test (CMT) } \\
\hline Learning (CMT-L) & $1500 s^{b}$ & $z=2.686, p=0.047^{\#}$ & $720 s^{k}$ & $z=-0.806, p=0.461^{\#}$ & $1380 s^{b}$ & $z=2.188, p=0.091^{\#}$ \\
\hline Recall (CMT-R) & $17.44 s^{\mathrm{C}}$ & $z=-1.706, p=0.139^{\#}$ & $38.72 \mathrm{~s}^{\mathrm{i}}$ & $z=-1.340, p=0.436^{\#}$ & $19.27 s^{c}$ & $z=-1.563, p=0.169^{\#}$ \\
\hline \multicolumn{7}{|c|}{ Navigational tasks in real environment } \\
\hline Landmark recognition & $15^{9}$ & $z=0.202, p=0.461^{\#}$ & & & & \\
\hline Route strategy & $7^{\mathrm{h}}$ & & & & & \\
\hline Throwback & $6 / 6^{i}$ & & & & & \\
\hline Map-following task & $3 / 4^{j}$ & $z=-0.827, p=0.342^{\#}$ & $4 / 4^{j}$ & & $4 / 4^{j}$ & \\
\hline
\end{tabular}

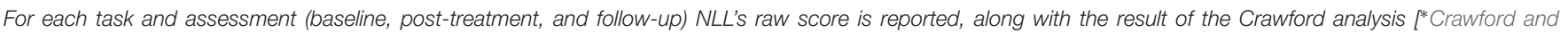

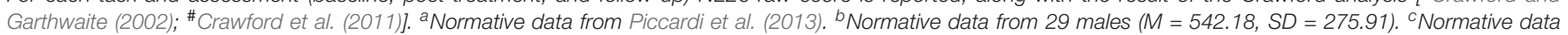

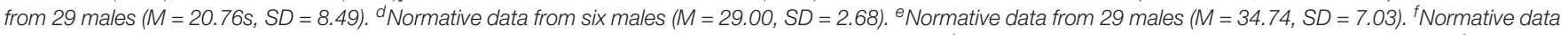

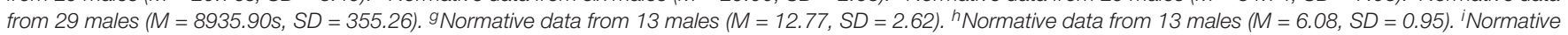

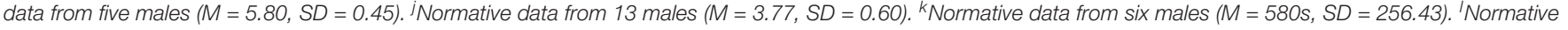
data from six males $(M=21.19 \mathrm{~s}, S D=8.66)$.

alternate version of the map-following task was errorless (four out of four segments correctly executed), with an effectiveness of $100 \%$. Improvement was stable 8 months later (Table 2).

Interestingly, after IBT NLL performed within the normal range on ecological memory tests (RBMT-3) in which he initially performed below the cut-offs (effectiveness on General Memory Index $=32.81 \%$ ), suggesting that improvement on navigational skills also generalized to episodic memory (Table 3 ).

Immediately after the IBT, NLL also performed at ceiling on the Object Generation and Color subtests of the CVMIB, with an effectiveness of $100 \%$ in both subtests.

\section{DISCUSSION}

When NLL came to our observation he was seriously worried about his topographical disorientation and memory impairment. At baseline, he showed deficits in topographical and visuospatial learning and an inability to form a cognitive map of environmental space. We also found a specific deficit in generating mental images of objects and in maintaining them. Performances on landmark recognition, route learning, and mapfollowing tasks were well within the normal range, even if they were performed with evident efforts. Neuropsychological evaluation highlighted a deficit in learning and retrieving
TABLE 3 | Rivermead Behavioral Memory Test-3.

\begin{tabular}{lcc}
\hline Subtest & $\begin{array}{c}\text { NLL's score } \\
\text { (baseline) }\end{array}$ & $\begin{array}{c}\text { NLL's score } \\
\text { (post-treatment) }\end{array}$ \\
\hline Names & 6 & 7 \\
Belongings & 6 & 8 \\
Appointments & 4 & 4 \\
Picture recognition & 14 & 14 \\
Story recall - immediate & $\mathbf{5 . 5}$ & 9 \\
Story recall - delayed & $\mathbf{4 . 5}$ & 8.5 \\
Face recognition - delayed & 14 & 10 \\
Route recall - immediate & 13 & 13 \\
Route recall - delayed & $\mathbf{8}$ & 13 \\
Messages - immediate & 6 & 6 \\
Messages - delayed & 6 & 6 \\
Orientation and date & 14 & 13 \\
Novel task - immediate & $\mathbf{1 8}$ & 48 \\
Novel task - delayed & $\mathbf{6}$ & 17 \\
GMl & 83 & 104 \\
\hline Pathological scores & &
\end{tabular}

Pathological scores are marked in bold. GMI, general memory index.

structured verbal material (i.e., stories), despite an intact ability to learn and recall unstructured verbal material, (i.e., word lists). IBT yielded a significant improvement in all the areas 
TABLE 4 | Complete visual mental imagery battery at baseline.

\begin{tabular}{|c|c|c|c|c|c|c|c|}
\hline \multirow{2}{*}{$\begin{array}{l}\text { Process } \\
\text { Generation }\end{array}$} & \multirow{2}{*}{$\begin{array}{l}\text { Subtest } \\
\text { Buildings }\end{array}$} & \multirow{2}{*}{$\begin{array}{c}\text { Maximum } \\
\text { score }\end{array}$} & \multicolumn{2}{|c|}{$\begin{array}{c}\text { Control } \\
\text { (Mean and SD) }^{\mathrm{a}}\end{array}$} & \multirow{2}{*}{$\begin{array}{c}\text { NLL's score } \\
\text { (baseline) } \\
18\end{array}$} & \multicolumn{2}{|c|}{$\begin{array}{c}\text { Crawford analyses } \\
\qquad(t \text { and } p)\end{array}$} \\
\hline & & & 19.28 & 1.04 & & -1.209 & 0.119 \\
\hline & Objects & 20 & 19.60 & 0.78 & 18 & -2.016 & 0.027 \\
\hline \multirow[t]{3}{*}{ Maintenance } & Color 1 & 10 & 9.57 & 0.57 & 10 & 0.741 & 0.233 \\
\hline & Color 2 & 10 & 9.82 & 0.39 & 9 & -2.066 & 0.024 \\
\hline & Color 3 & 10 & 9.57 & 0.74 & 9 & -0.757 & 0.228 \\
\hline \multirow[t]{2}{*}{ Inspection } & Objects & 20 & 18.67 & 1.18 & 18 & -0.558 & 0.291 \\
\hline & Letters & 20 & 19.50 & 1.10 & 20 & 0.447 & 0.329 \\
\hline \multirow[t]{2}{*}{ Transformation } & Folding & 20 & 16.75 & 2.93 & 12 & -1.593 & 0.062 \\
\hline & Mental rotation & 20 & 16.71 & 2.66 & 14 & -1.001 & 0.163 \\
\hline
\end{tabular}

a Normative data are derived from Palermo et al. (2016).

in which NLL was impaired. Indeed, after 8 weeks of IBT, NLL's performances did not differ from controls' ones in visuo-spatial and topographical learning, suggesting that IBT fostered memory for positions within both reaching and vista space; he also performed without differences from controls on CMT, suggesting that NLL recovered the ability to form a cognitive map of the environmental space. Accordingly, after IBT he ameliorated his ability to use a map for navigating the environmental space, performing at ceiling on the map-following task. Interestingly, also episodic memory improved over the original performance: after IBT NLL's performances on learning and recalling a story fell well within the normal range. These results deserve great attention in the light of possible clinical applications to topographical disorientation and episodic memory deficit as well as for their theoretical implications.

Despite the single case methodology, present findings provide a unique Contribution To The Field of neuropsychological rehabilitation of topographical disorientation. To the best of our knowledge, there is no evidence about effective neuropsychological rehabilitation of topographical disorientation. The only papers describing the rehabilitation of this disorder, indeed, focused on the acquisition of compensatory strategies (Incoccia et al., 2009; Bouwmeester et al., 2015; Svoboda et al., 2018); in some cases (Svoboda et al., 2018), the intervention was just aimed to allow patients to autonomously navigate in very familiar environments; in others, no generalization occurred for untreated materials (Davis and Coltheart, 1999). However, topographical disorientation may have a great impact on individual functioning. As it happened for NLL, people with topographical disorientation experience great difficulties which may prevent full recovery of autonomies. Before IBT, NLL used compensatory non-spatial strategies to cope with his daily life activities and professional duties, which likely resulted in non-pathological performances on ecological assessment of navigational skills (i.e., performance on landmark recognition, route learning, and map-following tasks). However, the compensation did not fully prevent NLL from getting lost and experiencing daily life difficulties. Indeed, he failed in processing and acquiring proper spatial information, such as positions, as also demonstrated by pathological scores on
WalCT. After IBT, NLL was able to learn and recall spatial positions within the environmental, vista, and reaching spaces, suggesting that the neuropsychological rehabilitation protocol we developed was able to restore the spatial mechanisms disrupted by traumatic brain injury.

Spatial information is processed by a redundant code in the brain and the interaction between different areas is crucial for successfully navigating within the environmental space (Ekstrom et al., 2017). IBT likely taps on the wide network of areas involved in generating mental images of environmental space, including the hippocampus, the retrosplenial cortex and the parahippocampal place area (Boccia et al., 2015, 2017b). Considering that spatial navigation and mental imagery of familiar places arise from the interaction between these brain areas (Boccia et al., 2016, 2017b, 2019) it is possible that IBT, fostering the interaction between these regions, allowed to restore mechanisms of environmental navigation on account of the redundant code within this network (Boccia et al., 2019). This interpretation is consistent with lesion location and extension of NLL, which mainly involved the right temporal lobe, sparing other nodes of the parieto-temporo medial network of spatial navigation (Boccia et al., 2017a).

Besides the severe topographical disorientation, NLL was also affected by a severe episodic memory deficit. Interestingly, the effect of IBT generalized to episodic memory. Previous evidence supports the use of imagery-based trainings for neuropsychological rehabilitation of memory (Piras et al., 2011). Most randomized control trials (RCTs) used visual imagery and visualization of stories (Chiaravalloti et al., 2005) of relevant everyday materials (Kaschel et al., 2002). Here we found that imagery-based training of spatial abilities may improve performance on the recall of a story. Also, NLL reported that recall of relevant everyday events was significantly improved after IBT. He said “... now I'm able to tell my father what happened during the day when he calls me in the evening." This result ties well with the recent hypothesis that mechanisms of planning and memory have their phylogenetic roots within mechanisms of spatial navigation in the physical world (Buzsaki and Moser, 2013). According to this view episodic memory 
has evolved from mechanisms of environmental navigation, and shares the same neuronal algorithms used to navigate within the real environment. In this vein, the improvement we found for episodic memory may be due to the restoring of imbalanced spatial "root" mechanisms. Also, this result is consistent with the idea that "spatial navigation serves as a model system to identify key coding principles governing cognitive spaces" (Bellmund et al., 2018, p. 362).

An alternative explanation for NLL's disabilities is related to his visual imagery deficits: a lesion in the right temporal region may cause the slight deficit we observed at baseline in generating and maintaining mental images; accordingly, NLL complained of a deficit in imaging landmarks and routes. This deficit might have caused a mixed spatial and episodic memory deficit. In this light, the recovery of spatial and episodic memory deficits may be mediated by the recovery of mental imagery skills we detected at the post-treatment evaluation. However, it has to be noted that we observed only a slight imagery deficit at baseline, in contrast to NLL's dramatic disability in navigating within highly familiar and novel environments and in remembering his own life events.

Even if compelling, present results deserve caution, since the effects we detected are based on the observation of a single case. Thus, further systematic investigations of imagery-based rehabilitation protocols for spatial navigation and memory (especially by means of RCTs) are needed to draw definite conclusions.

\section{DATA AVAILABILITY}

The datasets generated for this study are available on request to the corresponding author.

\section{ETHICS STATEMENT}

The studies involving human participants were reviewed and approved by Local ethical committee of the IRCCS Fondazione

\section{REFERENCES}

Aguirre, G. K., and D’Esposito, M. D. (1999). Topographical disorientation: a synthesis and taxonomy. Brain 122, 1613-1628. doi: 10.1093/brain/122.9.1613

Bellmund, J. L. S., Gardenfors, P., Moser, E. I., and Doeller, C. F. (2018). Navigating cognition: spatial codes for human thinking. Science 362:eaat6766. doi: 10.1126/ science.aat6766

Bianchini, F., Incoccia, C., Palermo, L., Piccardi, L., Zompanti, L., Sabatini, U., et al. (2010). Developmental topographical disorientation in a healthy subject. Neuropsychologia 48, 1563-1573. doi: 10.1016/j.neuropsychologia.2010.01.025

Bianchini, F., Palermo, L., Piccardi, L., Incoccia, C., Nemmi, F., Sabatini, U., et al. (2014). Where Am I? A new case of developmental topographical disorientation. J. Neuropsychol. 8, 107-124. doi: 10.1111/jnp.12007

Boccia, M., Guariglia, C., Sabatini, U., and Nemmi, F. (2016). Navigating toward a novel environment from a route or survey perspective: neural correlates and context-dependent connectivity. Brain Struct. Funct. 221, 2005-2021. doi: 10.1007/s00429-015-1021-z

Boccia, M., Nemmi, F., and Guariglia, C. (2014). Neuropsychology of environmental navigation in humans: review and meta-analysis of fMRI studies in healthy participants. Neuropsychol. Rev. 24, 236-251. doi: 10.1007/s11065014-9247-8
Santa Lucia in Rome. The patients/participants provided their written informed consent to participate in this study. Written informed consent was obtained from the individual(s) for the publication of any potentially identifiable images or data included in this article.

\section{AUTHOR CONTRIBUTIONS}

All the authors significantly contributed to the work, read, and approved the final version of the manuscript. AD and MPC performed the neuropsychological assessment. $\mathrm{MB}, \mathrm{SD}, \mathrm{AD}$, and CG conceived the IBT. SD and AB conducted the IBT. MB and $A B$ ran the statistical analyses and wrote the first draft of the manuscript.

\section{FUNDING}

This work was partially supported by funding from the Italian Ministry of Health to the IRCCS Fondazione Santa Lucia (Ricerca Corrente).

\section{ACKNOWLEDGMENTS}

We are thankful to Giuseppe Iaria and NeuroLab (www.neurolab. ca) for providing us with the Cognitive Map Test (CMT). We also wish to thank Alice Teghil who revised the final draft of the manuscript.

\section{SUPPLEMENTARY MATERIAL}

The Supplementary Material for this article can be found online at: https://www.frontiersin.org/articles/10.3389/fnhum. 2019.00322/full\#supplementary-material

Boccia, M., Piccardi, L., Palermo, L., Nemmi, F., Sulpizio, V., Galati, G., et al. (2015). A penny for your thoughts! patterns of fMRI activity reveal the content and the spatial topography of visual mental images. Hum. Brain Mapp. 36, 945-958. doi: 10.1002/hbm.22678

Boccia, M., Sulpizio, V., Nemmi, F., Guariglia, C., and Galati, G. (2017a). Direct and indirect parieto-medial temporal pathways for spatial navigation in humans: evidence from resting-state functional connectivity. Brain Struct. Funct. 222, 1945-1957. doi: 10.1007/s00429-016-1318-6

Boccia, M., Sulpizio, V., Palermo, L., Piccardi, L., Guariglia, C., and Galati, G. (2017b). I can see where you would be: patterns of fMRI activity reveal imagined landmarks. Neuroimage 144, 174-182. doi: 10.1016/j.neuroimage.2016. 08.034

Boccia, M., Sulpizio, V., Teghil, A., Palermo, L., Piccardi, L., Galati, G., et al. (2019). The dynamic contribution of the high-level visual cortex to imagery and perception. Hum. Brain Mapp. 40, 2449-2463. doi: 10.1002/hbm.24535

Bouwmeester, L., Van De Wege, A., Haaxma, R., and Snoek, J. W. (2015). Rehabilitation in a complex case of topographical disorientation. Neuropsychol. Rehabil. 25, 1-14. doi: 10.1080/09602011.2014.923318

Buzsaki, G., and Moser, E. I. (2013). Memory, navigation and theta rhythm in the hippocampal-entorhinal system. Nat. Neurosci. 16, 130-138. doi: 10.1038/nn. 3304 
Byrne, P., Becker, S., and Burgess, N. (2007). Remembering the past and imagining the future: a neural model of spatial memory and imagery. Psychol. Rev. 114, 340-375. doi: 10.1037/0033-295x.114.2.340

Chiaravalloti, N. D., Deluca, J., Moore, N. B., and Ricker, J. H. (2005). Treating learning impairments improves memory performance in multiple sclerosis: a randomized clinical trial. Mult. Scler. 11, 58-68. doi: 10.1191/ $1352458505 \mathrm{~ms} 1118 \mathrm{oa}$

Conson, M., Bianchini, F., Quarantelli, M., Boccia, M., Salzano, S., Di Vita, A., et al. (2018). Selective map-following navigation deficit: a new case of developmental topographical disorientation. J. Clin. Exp. Neuropsychol. 40, 940-950. doi: 10. 1080/13803395.2018.1451493

Corsi, P. M. (1972). Human Memory and the Medial Temporal Region of the Brain. Ph.D. thesis, McGill University, Montreal, QC.

Crawford, J. R., and Garthwaite, P. H. (2002). Investigation of the single case in neuropsychology: confidence limits on the abnormality of test scores and test score differences. Neuropsychologia 40, 1196-1208. doi: 10.1016/s00283932(01)00224-x

Crawford, J. R., Garthwaite, P. H., and Ryan, K. (2011). Comparing a single case to a control sample: testing for neuropsychological deficits and dissociations in the presence of covariates. Cortex 47, 1166-1178. doi: 10.1016/j.cortex.2011. 02.017

Davis, S. J. C., and Coltheart, M. (1999). Rehabilitation of topographical disorientation: an experimental single case study. Neuropsychol. Rehabil. 9, 1-30. doi: 10.1080/713755586

Ekstrom, A. D., Huffman, D. J., and Starrett, M. (2017). Interacting networks of brain regions underlie human spatial navigation: a review and novel synthesis of the literature. J. Neurophys. 118, 3328-3344. doi: 10.1152/jn.00531.2017

Ekstrom, A. D., Kahana, M. J., Caplan, J. B., Fields, T. A., Isham, E. A., Newman, E. L., et al. (2003). Cellular networks underlying human spatial navigation. Nature 425, 184-188. doi: 10.1038/nature01964

Holmes, G., and Horrax, G. (1919). Disturbances of spatial orientation and visual attention, with loss of stereoscopic vision. Arch. Neurol. Psychiatr. 1, 385-407.

Iaria, G., and Barton, J. J. (2010). Developmental topographical disorientation: a newly discovered cognitive disorder. Exp. Brain Res. 206, 189-196. doi: 10.1007/ s00221-010-2256-9

Iaria, G., Chen, J. K., Guariglia, C., Ptito, A., and Petrides, M. (2007). Retrosplenial and hippocampal brain regions in human navigation: complementary functional contributions to the formation and use of cognitive maps. Eur. J. Neurosci. 25, 890-899. doi: 10.1111/j.1460-9568.2007.05371.x

Incoccia, C., Magnotti, L., Iaria, G., Piccardi, L., and Guariglia, C. (2009). Topographical disorientation in a patient who never developed navigational skills: the (re)habilitation treatment. Neuropsychol. Rehabil. 19, 291-314. doi: 10.1080/09602010802188344

Kaschel, R., Della Sala, S., Cantagallo, A., Fahlböck, A., Laaksonen, R., and Kazen, M. (2002). Imagery mnemonics for the rehabilitation of memory: a randomised group controlled trial. Neuropsychol. Rehabil. 12, 127-153. doi: 10.1080/09602010143000211

Kravitz, D. J., Saleem, K. S., Baker, C. I., and Mishkin, M. (2011). A new neural framework for visuospatial processing. Nat. Rev. Neurosci. 12, 217-230. doi: 10.1038/nrn3008

Levine, D. N., Warach, J., and Farah, M. (1985). Two visual systems in mental imagery: dissociation of "what" and "where" in imagery disorders due to bilateral posterior cerebral lesions. Neurology 35, 1010-1018.

Money, J., Alexander, D., and Walker, H. T. (1965). A Standardized Road-Map Test of Direction Sense; Manual. Baltimore: Johns Hopkins Press.

Montello, D. R. (1993). "Scale and multiple psychologies of space in spatial information theory: a theoretical basis for GIS," in Proceedings of the COSIT: European Conference on Spatial Information Theory (Berlin: Springer-Verlag), 312-321. doi: 10.1007/3-540-57207-4_21

O'Keefe, J., and Dostrovsky, J. (1971). The hippocampus as a spatial map. Preliminary evidence from unit activity in the freely-moving rat. Brain Res. 34, 171-175. doi: 10.1016/0006-8993(71)90358-1

Palermo, L., Piccardi, L., Bianchini, F., Nemmi, F., Giorgio, V., Incoccia, C., et al. (2014). Looking for the compass in a case of developmental topographical disorientation: a behavioral and neuroimaging study. J. Clin. Exp. Neuropsychol. 36, 464-481. doi: 10.1080/13803395.2014.904843
Palermo, L., Piccardi, L., Nori, R., Giusberti, F., and Guariglia, C. (2016). The impact of ageing and gender on visual mental imagery processes: a study of performance on tasks from the complete visual mental imagery battery (CVMIB). J. Clin. Exp. Neuropsychol. 38, 752-763. doi: 10.1080/13803395.2016. 1161735

Piccardi, L., Bianchini, F., Argento, O., De Nigris, A., Maialetti, A., Palermo, L., et al. (2013). The Walking Corsi Test (WalCT): standardization of the topographical memory test in an Italian population. Neurol. Sci. 34, 971-978. doi: 10.1007/s10072-012-1175-x

Piccardi, L., Iaria, G., Ricci, M., Bianchini, F., Zompanti, L., and Guariglia, C. (2008). Walking in the Corsi test: which type of memory do you need? Neurosci. Lett. 432, 127-131. doi: 10.1016/j.neulet.2007.12.044

Piras, F., Borella, E., Incoccia, C., and Carlesimo, G. A. (2011). Evidence-based practice recommendations for memory rehabilitation. Eur. J. Phys. Rehabil. Med. 47:27.

Siegel, A. W., and White, S. H. (1975). The Development of Spatial Representations of Large-Scale Environments. Amsterdam: Academic Press.

Stark, M., Coslett, H. B., and Saffran, E. M. (1996). Impairment of an egocentric map of locations: implications for perception and action. Cogn. Neuropsychol. 13, 481-523.

Sulpizio, V., Boccia, M., Guariglia, C., and Galati, G. (2017). Implicit coding of location and direction in a familiar, real-world "vista" space. Behav. Brain Res. 319, 16-24. doi: 10.1016/j.bbr.2016.10.052

Sulpizio, V., Boccia, M., Guariglia, C., and Galati, G. (2018). Neural codes for one's own position and direction in a real-world "vista" environment. Front. Hum. Neurosci. 12:167.

Sulpizio, V., Committeri, G., and Galati, G. (2014). Distributed cognitive maps reflecting real distances between places and views in the human brain. Front. Hum. Neurosci. 8:716. doi: 10.3389/fnhum.2014.00716

Svoboda, E., Mccarthy, J., and Moscovitch, M. (2018). A case study of topographical disorientation: behavioural intervention for achieving independent navigation AU - Rivest, Josée. Neuropsychol. Rehabil. 28, 797-817. doi: 10.1080/09602011. 2016.1160833

Takahashi, N., Kawamura, M., Shiota, J., Kasahata, N., and Hirayama, K. (1997). Pure topographic disorientation due to right retrosplenial lesion. Neurology 49, 464-469. doi: 10.1212/wnl.49.2.464

Taube, J. S. (1998). Head direction cells and the neurophysiological basis for a sense of direction. Prog. Neurobiol. 55, 225-256. doi: 10.1016/s0301-0082(98)00 004-5

Thurstone, L. L. (1937). Ability, motivation, and speed. Psychometrika 2, 249-254. doi: $10.1007 /$ bf02287896

Vass, L. K., and Epstein, R. A. (2013). Abstract representations of location and facing direction in the human brain. J. Neurosci. 33, 6133-6142. doi: 10.1523/ JNEUROSCI.3873-12.2013

Wang, R. F., and Spelke, E. S. (2002). Human spatial representation: insights from animals. Trends Cogn. Sci. 6, 376-382. doi: 10.1016/s1364-6613(02)01961-7

Wilson, B. A., Greenfield, E., Clare, L., Baddeley, A., Cockburn, J., Watson, P., et al. (2008). Rivermead Behavioral Memory Test, 3rd Edn. London: Thames Valley Test Company.

Wolbers, T., and Hegarty, M. (2010). What determines our navigational abilities? Trends Cogn. Sci. 14, 138-146. doi: 10.1016/j.tics.2010.01.001

Wolbers, T., and Wiener, J. M. (2014). Challenges for identifying the neural mechanisms that support spatial navigation: the impact of spatial scale. Front. Hum. Neurosci. 8:571. doi: 10.3389/fnhum.2014.00571

Conflict of Interest Statement: The authors declare that the research was conducted in the absence of any commercial or financial relationships that could be construed as a potential conflict of interest.

Copyright (c) 2019 Boccia, Bonavita, Diana, Di Vita, Ciurli and Guariglia. This is an open-access article distributed under the terms of the Creative Commons Attribution License (CC BY). The use, distribution or reproduction in other forums is permitted, provided the original author(s) and the copyright owner(s) are credited and that the original publication in this journal is cited, in accordance with accepted academic practice. No use, distribution or reproduction is permitted which does not comply with these terms. 